\title{
Chapter 19 \\ Understanding Complexity in a Practical Combinatorial Problem Using Mathematical Programming and Constraint Programming
}

\author{
Beatriz B. Oliveira and Maria Antónia Carravilla
}

\begin{abstract}
Optimization problems that are motivated by real-world settings are often complex to solve. Bridging the gap between theory and practice in this field starts by understanding the causes of complexity of each problem and measuring its impact in order to make better decisions on approaches and methods. The Job-Shop Scheduling Problem (JSSP) is a well-known complex combinatorial problem with several industrial applications. This problem is used to analyse what makes some instances difficult to solve for a commonly used solution approach - Mathematical Integer Programming (MIP) - and to compare the power of an alternative approach: Constraint Programming (CP). The causes of complexity are analysed and compared for both approaches and a measure of MIP complexity is proposed, based on the concept of load per machine. Also, the impact of problem-specific global constraints in CP modelling is analysed, making proof of the industrial practical interest of commercially available CP models for the JSSP.
\end{abstract}

Keywords Job-shop scheduling problem $\cdot$ Mathematical programming Constraint programming $\cdot$ Global constraints $\cdot$ Complexity

\subsection{Introduction}

The Job-Shop Scheduling Problem (JSSP) and its many extensions and variations have been thoroughly studied on the field of Optimization, due to its computational difficulty. This problem is NP-hard [8] and was even qualified as "one of the most computationally stubborn combinatorial problems" [3]. For these reasons, it will be analysed in this paper as a starting point to understand the meaning of complexity for both approaches: Mathematical Programming and Constraint Programming.

B. B. Oliveira $(\bowtie) \cdot$ M. A. Carravilla

INESC TEC and Faculty of Engineering, University of Porto, Porto, Portugal

e-mail: beatriz.oliveira@fe.up.pt

M. A. Carravilla

e-mail: mac@fe.up.pt 
The JSSP summarily consists of scheduling $J$ jobs to be processed in $M$ machines with the goal of minimizing the time it takes for all the operations to end (makespan). Each job takes a fixed processing time in each machine and there is a certain sequence of machines that it should follow - each job has its own specific sequence. The operations must be completed - once a job starts on a specific machine, it must take the full processing time. Also, a machine can only handle one job at the time.

The JSSP has numerous applications in the industry, namely on production planning and scheduling. In the past years, several extensions and adaptations are arising in the literature $[9,12]$, many concerning real applications.

This paper has two main objectives. Firstly, it aims to gain some insights regarding the factors that induce complexity on the Mathematical Programming approach to this problem. As this approach is commonly used to solve real-world problems, understanding these factors can help practitioners identify beforehand the challenges and/or opportunities that arise from the characteristics of the problem and therefore make better decisions regarding e.g. solution methods.

Secondly, it aims to understand whether the alternative approach of Constraint Programming (CP) is useful to tackle to the "difficult to solve" instances. Above all, there is the goal to understand the power of the commercially available CP models for the JSSP and, consequently, whether they are a realistic solid alternative for practitioners.

The paper is thus divided into two main sections. The first section is related to the Mathematical Programming approach. It presents a mixed-integer formulation for this combinatorial problem that is commonly used in the literature and assesses its computational power with eighty-two literature instances. The main objective is to understand which factors are more relevant to explain the complexity and "stubbornness" of the JSSP, namely to understand the impact of size. Therefore, on Sect. 19.2.1, the MIP model is presented; the computational results are discussed on Sect. 19.2.2. In this section, a potential measure of instance complexity is also presented and tested. The second part is devoted to the Constraint Programming approach to the JSSP (Sect. 19.3). Here, three different CP formulations are presented (Sect. 19.3.1) and tested against the performance of the MIP model (Sect.19.3.2). One of the CP formulations is a model commercially available, including global constraints specifically developed for this problem. The main goal is herein to understand the power and limitations of CP programs, namely the commercially available one. Finally, on Sect. 19.4 the main conclusions are drawn.

The overall purpose of this paper is to draw attention to the impact of understanding and measuring impact when tackling specially complex problems, such as real-world applications of the JSSP. This purpose is materialised in the following contributions:

- A quantitative-based discussion on factors that induce complexity for a MIP approach to the JSSP, namely the total size of the instance and relationship between number of jobs and machines;

- A proposed measure of MIP-complexity of JSSP instances based on load per machine; 
- A comparison of the computational power of different CP formulations for the $J S S P$, with insights related with commercially available CP models, which show significant advantages when tackling complex instances;

- A comparison between CP and MIP approaches to the JSSP, establishing their advantages and shortcomings;

- A quantitative evidence of the benefits of using commercially available CP models to tackle complex JSSP instances and settings.

\subsection{Mathematical Programming Approach}

\subsubsection{MIP Model}

This model follows the one proposed by Applegate and Cook [3], based on the disjunctive programming problem presented in Balas [5].

\section{Sets and indexes}

$$
\begin{gathered}
\mathscr{J} \text { set of jobs }(|\mathscr{J}|=J) \\
\mathscr{M} \text { set of machines }|\mathscr{M}|=M) \\
i, j \in \mathscr{J} \text { index of jobs }(i, j=\{1, \ldots, J\}) \\
k \in \mathscr{M} \text { index of machines }(k=\{1, \ldots, M\})
\end{gathered}
$$

\section{Parameters}

$$
\begin{gathered}
p_{i k} \text { the } k \text { th operation (machine) for job } i \\
t_{i k} \text { processing time of job } i \text { on machine } k \\
L \text { significantly large value }
\end{gathered}
$$

\section{Decision Variables}

$x_{i k}$ starting time of job $i$ on machine $k$

$y_{i j k}(=1)$ if job $i$ is scheduled before job $j$ in machine $k$ $c$ makespan

\section{Mathematical Program}

$$
\begin{array}{rlrl}
\min c & & \\
\text { s.t. } \quad x_{i p_{i k}} & \geq x_{i p_{i k-1}}+t_{i p_{i k-1}}, & & \forall i \in \mathscr{J}, \forall k \in \mathscr{M} \backslash\{1\} \\
x_{i k} & \geq x_{j k}+t_{j k}-L y_{i j k}, & & \forall k \in \mathscr{M}, \forall i, j>i \in \mathscr{J} \\
x_{j k} & \geq x_{i k}+t_{i k}-L\left(1-y_{i j k}\right), & & \forall k \in \mathscr{M}, \forall i, j>i \in \mathscr{J} \\
c & \geq x_{i p_{i M}}+t_{i p_{i M}} & & \forall i \in \mathscr{J} \\
x_{i k}, c & \geq 0, & & \forall i \in \mathscr{J}, k \in \mathscr{M} \\
y_{i j k} & \in\{0,1\}, & & \forall i, j>i \in \mathscr{J}, k \in \mathscr{M}
\end{array}
$$


The goal of this model is to minimize the total makespan (Eq. 19.1). The first constraint (Eq. 19.2) ensures that a job can only start to be processed in a machine if it has finished its processing on the previous machine (clearly, this does not apply to the first operation of the job).

The second and third constraints are related to the order of the jobs in each machine (Eqs. 19.3 and 19.4). They state that if a job precedes another job in a certain machine, then its starting time is not limited by the latter. However, if a job comes after another job in a machine, it can only start when this one has finished. The following constraint (Eq. 19.5) states that the total makespan is given by the job that finishes the processing last (i.e., based on the last machine: the $M$ th machine the job will go through).

Finally, the decision variable that sets the starting time of each job on each machine as well as the makespan should be non-negative, and the precedence variable is set as a binary (Eqs. 19.6 and 19.7).

\subsubsection{Computational Tests and Results}

In order to understand the behaviour of this MIP model, 82 instances from the literature were run. The computational tests were run on a Toshiba Personal Computer with 16 Gigabyte of RAM memory, and with a processor Intel(R) Core(TM) i7-4600U CPU @ 2.10 GHz 2.70GHz. The model was developed in a OPL Project in IBM ILOG CPLEX Optimization Studio 12.6.1.0 and the MIP Solver used was CPLEX. The time limit set for the resolution of each instance was $30 \mathrm{~min}$. The instances' characteristics and solution can be found on the Appendix (Table 19.4) and the main results are summarised on Table 19.1.

Table 19.1 Summary of the average results of the MIP model

\begin{tabular}{l|r|l|l|l|l|l}
\hline$J$ & \multicolumn{1}{|c|}{$M$} & \#instances & Avg. sol. time (s) & Sol. time std. dev. (s) & Avg. gap (\%) & Gap std. dev. \\
\hline 6 & 6 & 1 & 0 & 0 & 0 & 0 \\
\hline 10 & 5 & 5 & 13 & 18 & 0 & 0 \\
\hline 10 & 10 & 18 & 142 & 286 & 0 & 0 \\
\hline 15 & 5 & 5 & 1.800 & 0 & 29 & $5 \%$ \\
\hline 15 & 10 & 5 & 1.800 & 0 & 11 & $8 \%$ \\
\hline 15 & 15 & 5 & 1.800 & 0 & 12 & $3 \%$ \\
\hline 20 & 5 & 6 & 1.800 & 0 & 47 & $5 \%$ \\
\hline 20 & 10 & 10 & 1.800 & 0 & 39 & $9 \%$ \\
\hline 20 & 15 & 8 & 1.800 & 0 & 37 & $2 \%$ \\
\hline 20 & 20 & 4 & 1.800 & 0 & 22 & $4 \%$ \\
\hline 30 & 10 & 5 & 1.800 & 0 & 55 & $2 \%$ \\
\hline 50 & 10 & 10 & 1.800 & 0 & 76 & $3 \%$ \\
\hline
\end{tabular}




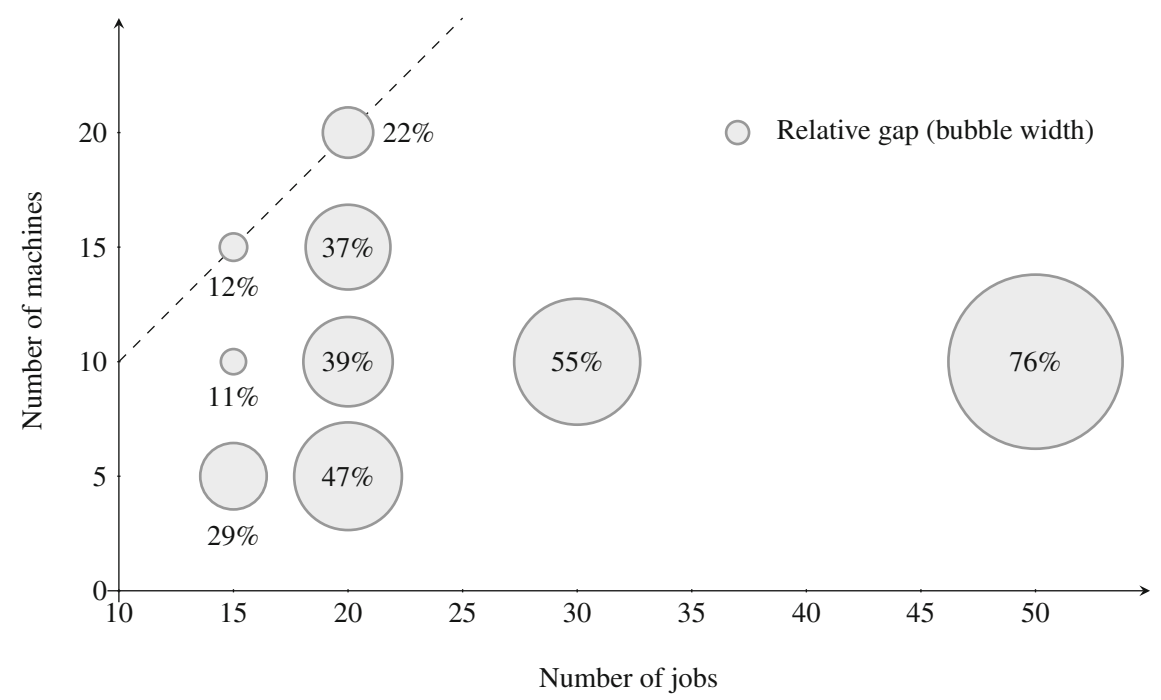

Fig. 19.1 Average MIP relative optimality gap attained per instance size

As it is possible to observe on Table 19.1, the solver was only able to prove optimality within the time limit $(30 \mathrm{~min})$ for the twenty-four smallest instances. ${ }^{1}$ The results are herein analysed for both resulting groups:

\section{Instances Solved to Optimality:}

For the smallest instances, solved to optimality, the average solution time increases with the size of the instances. Nevertheless, the magnitude of the variability of solution times for instances of the same size (given by its standard deviation, in Table 19.1) suggests that the size is not the only factor that translates into complexity and, consequently, to longer run times.

\section{Instances Not Solved to Optimality:}

For the fifty-eight instances that were not solved to optimality in $30 \mathrm{~min}$, the value of the optimality gap attained may shed some light on the matter of complexity. Firstly, it is interesting to compare the average results of the instances of type $(J, M)=(20,5)$ and of type $(J, M)=(15,15)$. The latter are bigger in terms of number of variables and constraints, yet the solver is able to achieve significantly lower gaps for them ( $47 \%$ vs. $12 \%$ ). This is another evidence that the "easiness to solve" is not only linked with the size of the problem.

Figure 19.1 represents the average gap attained for instances of the same size. The horizontal axis represents the number of jobs in the instances, the vertical axis represents the number of machines, and the width of the bubbles depicts the size of the average optimality gap. On an horizontal reading, it is possible to see that the optimality gap increases as instances tackle an increasing number of jobs. This may

\footnotetext{
${ }^{1}$ Instances ft06, ft10, abz5, abz6, la01-la05, la16-la20, orb01-orb10.
} 
be due to the fact that the number of jobs influences the most the size and thus, at some (limited) degree, the complexity of the model and its difficulty to solve to optimality. In fact, due to the structure of the binary variable $y_{i j k}, \forall i, j>i \in \mathscr{J}, k \in \mathscr{M}$, it can be seen that $J=|\mathscr{J}|$ has more impact on the number of variables that $M=|\mathscr{M}|$. Due to the structure of Constraints 19.3 and 19.4, it also strongly influences the number of constraints. In conclusion, this horizontal increase seems a natural consequence of the size of the instances, which is heavily dependent on the number of jobs $(J)$.

However, if one considers a fixed number of jobs (for example, $j=20$ on the horizontal axis) and analyses the impact of the number of machines in the optimality gap, the effect is reversed. It seems that, for a fixed number of jobs, the optimality gap achieved in the same run time is better for more balanced instances, with the same number of jobs and machines (even if that means a bigger number of variables and constraints).

Regarding the observable "easiness" to solve balanced instances (in terms of number of jobs and machines), one may consider several hypotheses. Please consider the following definition of optimality gap for a minimization problem: Gap = $(U B-L B) / L B$, where $L B$ stands for the lower bound, given by the best relaxed solution found on the Branch-and-Bound nodes not pruned at a time; and $U B$ stands for the upper bound, which is given by the best integer solution found so far. Due to the complexity of the methods and heuristics utilized by CPLEX to speed up the Branch-and-Bound procedure, it is difficult to understand how the lower bound evolves in different instances. Therefore, the main hypothesis presented to explain this behaviour is related with the update velocity of the upper bound. Analysing the data herein presented, it may be possible to say that the balanced structure (i.e., a similar number of jobs and machines) makes it easier for the solver to find admissible (integer) solutions and thus update the upper bound.

One could also argue that the effect of the increasing number of machines (that reduces the optimality gap for the same number of jobs) is not a matter of balance but of the existence of alternative admissible solutions. That is to say that it is not the balance between the two dimensions of the problem that is at stake but only the number of machines. In fact, if a certain problem is characterized by a big number of machines (and if the precedence of operations is not too restraining) it is possibly easier to find alternative optima, as well as admissible integer solutions that update the upper bound.

Other factors may influence the complexity of the instance, namely the precedences and processing times that may hinder or help the procedure to find admissible integer solutions, which may explain the high standard deviation of the solution times observed for the twenty-four smallest instances.

\section{A Measure of Complexity}

In order to further understand the concept of instance complexity, a measure of the load of solicitations applied to each machine throughout the scheduling horizon was designed. Dividing the horizon in partitions of the same size, the goal was to understand whether the load applied in the partitions (the need or demand of jobs 
to be scheduled there) was constant, and what was the maximum value it achieved throughout the horizon. So, for some partition $p$ of the horizon, the needs-to-capacity ratio $(r)$ is given by:

$$
r_{p}=\frac{\operatorname{count}\left(E S_{i k} \in p\right)}{M}, \forall i \in \mathscr{J}, \forall k \in \mathscr{M}
$$

Here, $M$ is, as before, the number of machines, and $E S_{i k}$ stands for the earliest start of job $i$ in machine $k$. The earliest start is defined by the precedence rules of each job:

$$
E S_{i k}=\sum_{m} t_{i m} \quad, \forall m \in \mathscr{M}: p_{i o}=m, p_{i o^{\prime}}=k, o<o^{\prime}
$$

Here, as before, $t_{i m}$ stands for the processing time of job $i$ on machine $m$ and $p_{i o}$ represents the $o$ th machine where job $i$ is processed. Therefore, the earliest start of a job on a machine is the result of the sum of the processing times of that same job on the preceding machines. For example, if job 1 can only be processed in machine $C$ after being processed in machines $A$ and $B$ then the earliest start of job 1 on machine $C$ is equal to the time it takes to be processed in machines $A$ and $B$. Therefore, the needs-to-capacity ratio is able to represent, at a certain extent, what are the needs of jobs to be scheduled in each machine, taking into consideration the precedences.

This measure was calculated for the eighty-two instances run. In order to adapt the partitions of the scheduling horizon to each instance, the length of the partitions was based on the instances' minimum value of the processing times:

$$
\text { length }=2 \cdot \min _{i k}\left(t_{i k}\right), \quad \forall i \in \mathscr{J}, \forall k \in \mathscr{M}
$$

Figure 19.2 represents the evolution of this ratio for instances $1 \mathrm{a} 10$ and $1 \mathrm{a} 37$. Both instances consider 15 jobs yet in 1 a 10 there are 5 machines whilst in 1 a 37 there are 15 machines. Despite the smaller size, the MIP solver achieved an optimality gap of $35 \%$ for the former (vs. $15 \%$ for the latter).

Fig. 19.2 Evolution of the needs-to-capacity ratio for the first 40 partitions of the scheduling horizon of instances 1 a10 and la37

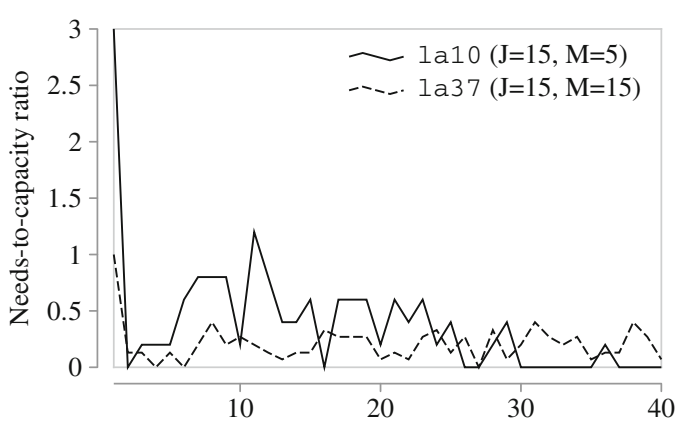


Fig. 19.3 Correlation between the optimality gap attained by the MIP solver and maximum needs-to-capacity ratio of the instances in Footnote 2

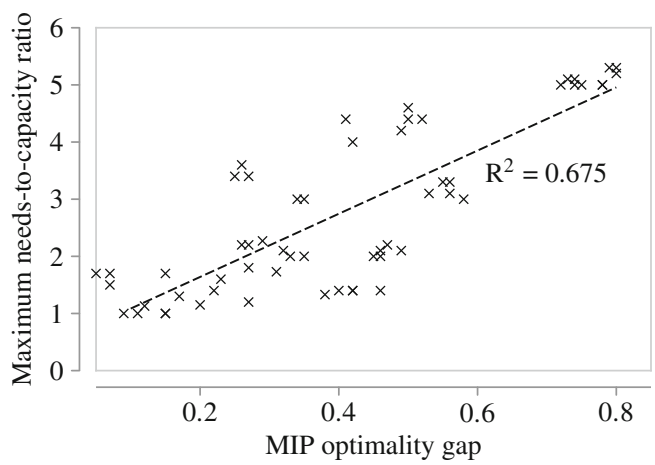

As explained before, the needs-to-capacity ratio represents, for each partition of time, the number of jobs whose earliest start in some machine falls within the partition, divided over the total number of machines. It is thus an average measure of the load per machine. Figure 19.2 is hence able to display the additional complexity of 1a10: the maximum and overall level of solicitations (needs) per machine is higher than in 1a37 and has more variation throughout time. This supports the statement that the precedences and processing times in the instance may hinder the solver to find admissible integer solutions and influence the solution time/quality more than the number of variables and constraints.

In order to compare instances in a easier way, the maximum ratio attained throughout the partitions of the scheduling horizon was calculated for each instance. Figure 19.3 represents the correlation between the maximum ratio and the optimality gap attained by the MIP solver (for those instances in which the solver was unable to prove optimality within the time limit ${ }^{2}$ ). Table 19.5 in the Appendix also presents these metrics, together with the quartile in which each maximum ratio falls when comparing the maximum needs-to-capacity ratio of the eighty-two instances. This table also contains information regarding the number of partitions, their length, and the standard deviation of the ratio throughout the horizon.

Figure 19.3 represents each instance by its optimality gap and maximum needs-tocapacity ratio. It is possible to observe that the ratio increases as the gap increases, i.e. as the MIP solver finds it more and more difficult to reach (or prove) optimality. The $R^{2}$ statistics with a value of $67 \%$ shows a good correlation between these two metrics, which supports the hypothesis that the ratio is a good indicator of an instance's complexity for the MIP solver. That is to say, the relation between the load of jobs that, at a certain point in time, are soliciting the machines and the capacity the system has to respond to these solicitations (i.e. the number of machines) has a direct influence on the difficulty the solver has to reach and prove optimality for a given instance.

\footnotetext{
${ }^{2}$ Instances in which the MIP solver was unable to prove optimality within the $30 \mathrm{~min}$ time limit: abz7-abz9, ft20, la06-la15, la21-1a40, swv01-swv20, yn1-yn4.
} 


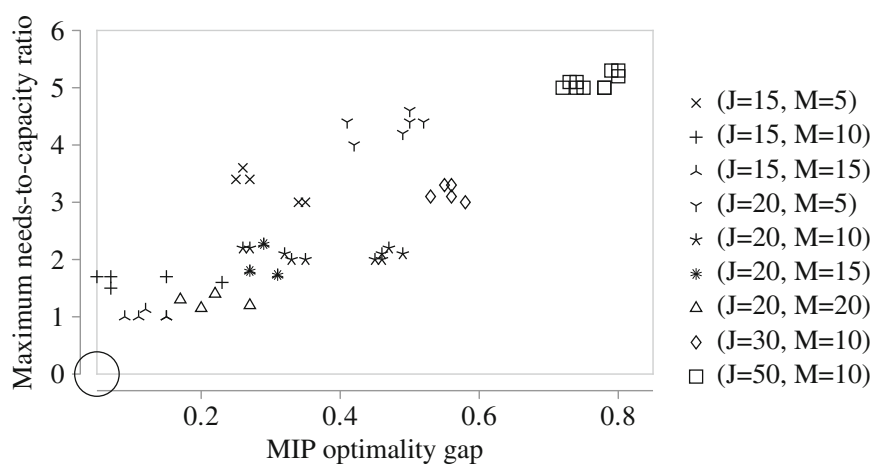

Fig. 19.4 Correlation between the optimality gap attained by the MIP solver and maximum needsto-capacity ratio of the previous instances, categorized by size (number of jobs $\mathrm{J}$ and number of machines M)

Figure 19.4 shows the size characteristics (number of jobs and machines) of the instances in Fig. 19.3. It is interesting to see from a different perspective the conclusion drawn before: an increase of the size does not imply an increase of complexity. At the same time, if one considers a specific group of instances of the same size, videlicet the instances with $(J, M)=(20,20)$ (triangle-shaped points), the maximum ratio attained is very similar amongst the instances while the optimality gap still shows some variation, not fully explained by the ratio (the dots representing the instances are spread horizontally on Fig. 19.4). This shows that are still other factors that may contribute to the complexity of the instance besides size and load of solicitations on the machines.

\subsection{Constraint Programming Approach}

"CP solving is based on intelligently enumerating all possible variable-value combinations" [13].

Constraint Programming is based on a flexible and intuitive modelling language, allowing for logic constraints, non-linear constraints, and almost every expression possible over the variables. It also requires that the domain of the variables (finite set of possible values) is set beforehand [13].

A CP program assigns values to variables without violating the constraints [4]. A search tree is built with several nodes, where each node is a solution, with some variables with a value already assigned to them. Each node then branches to several other nodes, each representing a similar solution but with other variable (from the remaining without assignment) allocated to some possible value. There are as many child nodes as there are possible values in the domain of the variable to assign [1]. The mechanism of constraint propagation consists on decreasing the domain of the 
variables not assigned yet due to the value assigned to a certain value in a iteration, and thus decreasing the number combinations to test [13].

Global constraints are a powerful element of CP programs. A global constraint is, by definition, a constraint that involves more than two variables. When designed to seize to the full potential the structure of the problem, these can be a powerful tool to facilitate constraint propagation and thus reduce significantly the search tree. It is important to recall that, although there are widely used global constraints (e.g. alldiff), some of the best performing global constraints that solve specially difficult problems are usually designed specifically for them. For the most studied problems in CP, global constraints have been designed and enhanced in the past years [10]. It is also important to note that in CP, unlike MIP problems, increasing the number of constraints may indeed be beneficial as it helps propagation.

Due to their characteristics, it is often the case that CP programs should perform well, by nature and theory, at finding admissible solutions, although only being able to prove optimality by explicitly searching all nodes and comparing their results. MIP solvers, on the other hand, although having more difficulty at finding admissible solutions, are able to prove optimality by setting upper and lower bounds and determining the optimality gap, i.e. it is not needed to explicitly explore the full solution space.

Scheduling problems are a classical application of CP due to their above-discussed "stubbornness". Therefore, specific well-performing global constraints have been designed and tested for it. Moreover, since it was previously inferred that the difficulty to find admissible integer solutions may be an important factor on the "stubbornness" of this problem (Sect. 19.2.2), Constraint Programming should be an appropriate approach to tackle this problem. So, in the next section, three different CP models are presented and tested. The first two are CP variations of the previously presented MIP model. The third one uses global constraints developed specifically for this problem and different decision variables.

\subsubsection{CP Models}

Three CP models are used in this paper to understand and compare the power of Constraint Programming models, namely and especially of global constraints.

The first model (CP1), is nearly a translation of the MIP model presented in Sect. 19.2.1. Since logic constraints can be used in CP, Constraints 19.3 and 19.4 (of the MIP model) can be translated into one disjunctive equation, and thus there is no need to use the auxiliary binary variables $y_{i j k}$. The second model (CP2) is a variation of CP1, where the makespan variable $(c)$ is eliminated (as well as Constraint 19.5). Since CP does not require its model elements to be linear, the objective function can be stated as the minimization of the maximum value amongst several, which was not possible using Mathematical Linear Programming. Finally, the third model (CP3) is based on the IBM ILOG CPLEX Optimization Studio example sched_jobshop for 
CP and seizes all the advantages of Constraint Programming: the decision variables' representation is more appropriate and two specifically developed global constraints are used.

\subsubsection{CP1}

Using the same sets, indexes and parameters of the MIP Model presented:

\section{Decision Variables}

$x_{i k} \in\{0, \ldots, 10000\}$ and integer starting time of job $i$ on machine $k$

$c \in\{0, \ldots, 10000\}$ and integer makespan

\section{CP1 Model}

$$
\min c
$$

s.t. $\quad x_{i p_{i k}} \geq x_{i p_{i k-1}}+t_{i p_{i k-1}}$,

$$
\forall i \in \mathscr{J}, \forall k \in \mathscr{M} \backslash\{1\}
$$$$
x_{i k} \geq x_{j k}+t_{j k} \quad \vee \quad x_{j k} \geq x_{i k}+t_{i k} \text {, }
$$$$
\forall k \in \mathscr{M}, \forall i, j>i \in \mathscr{J}
$$

$$
c \geq x_{i p_{i M}}+t_{i p_{i M}}
$$$$
\forall i \in \mathscr{J}
$$

The main difference of this model (vs. the MIP model) is the absence of the $y_{i j k}$ variables and the presence of the disjunctive Constraint 19.10. In fact, CP models allow for this kind of logic constraints, which state that either the inequality on the left or the inequality of the right must hold. That is to say that for each machine either job $i$ waits for job $j$ to end (if it is scheduled after it) or the other way around. This type of constraint allows for this disjunction "or-or" to be stated without the auxiliary binary variable that states whether or not job $i$ precedes job $j$.

\subsubsection{CP2}

Using the same sets, indexes and parameters of the MIP Model presented:

\section{Decision Variables}

$x_{i k} \in\{0, \ldots, 10000\}$ and integer starting time of job $i$ on machine $k$

\section{CP2 Model}

$$
\begin{array}{rlrl} 
& \min \max _{i}\left\{x_{i p_{i M}}+t_{i p_{i M}}\right\} & \\
\text { s.t. } & x_{i p_{i k}} \geq x_{i p_{i k-1}}+t_{i p_{i k-1}}, & & \forall i \in \mathscr{J}, \forall k \in \mathscr{M} \backslash\{1\} \\
& x_{i k} \geq x_{j k}+t_{j k} \vee x_{j k} \geq x_{i k}+t_{i k}, & & \forall k \in \mathscr{M}, \forall i, j>i \in \mathscr{J}
\end{array}
$$


When comparing with CP1, this model has a different objective function and, consequently, the variable $c$ and the Constraint 19.11 are no longer needed. This is possible because CP allows for the use of non-linear equations, such as Eq. 19.12.

\subsubsection{CP3}

Using the same sets, and indexes of the MIP Model presented:

\section{Parameters}

$O_{i k}:(m, t)$ operation of job $i$ in the $k$ th machine, represented by a tuple of the machine in question $(m)$ and the associated processing time $(t)$

\section{Decision Variables}

$w_{i k}, \operatorname{size} O_{i k}: t$ interval that represents the operation of job $i$ on machine $k$, which must have the length (size) of the processing time $t$ of operation $O_{i k}$ $s_{k}$ sequence of the above-defined intervals (jobs) on machine $k$; built from the intervals for all $i$ jobs and $o$ machines such that $O_{i o}: m=k$

\section{CP3 Model}

$$
\begin{array}{rll}
\min & \max _{i}\left\{\operatorname{endOf}\left(w_{i M}\right)\right\} & \\
\text { s.t. } & \operatorname{noOverlap}\left(s_{k}\right) & \forall k \in \mathscr{M} \\
& \text { endBeforeStart }\left(w_{i k}, w_{i k+1}\right) & \forall i \in \mathscr{J}, \forall k \in \mathscr{M} \backslash\{M\}
\end{array}
$$

This model is considerably different from the previous two (and, consequently, from the MIP Model) mainly because the decision variables were changed in order to enable the use of two global constraints (Constraints 19.16 and 19.17), which were designed specifically for the JSSP and are able to fulfil the Constraint Programming potential to its maximum in this type of problems. In fact, our variables are now intervals, representing the length of the job operations by its size (which is a parameter) and their positioning by its start time (which is the decision variable) and finish time, and sequences of intervals, which represent the job scheduling in each machine. In fact, although the second decision variable is somehow repeating what the first one already says, it is needed in order to build Constraint 19.16, which will bring significant computational advantages.

The objective function is similar to the one on $\mathrm{CP} 2$, and attempts to minimize the makespan, i.e. the last interval or operation to end. Constraint 19.16 uses the structure of the decision variable sequence $s$, and ensures that there is no overlap of jobs in every machine. It has the same function as Constraints 19.3 and 19.4 in 
the MIP Model. Constraint 19.17 ensures that a job only starts on a machine after it has finished on the previous. It has the same function as Constraint 19.2 in the MIP Model.

\subsubsection{Computational Tests and Results}

In order to understand the behaviour and performance of these CP models versus the MIP model, eleven instances were chosen from the eighty-two tested for the Mathematical Programming approach (Sect. 19.2.2). These instances were chosen based on their performance with the MIP Model. For each size of instance (number of jobs and number of machines), the hardest instance to solve with Mathematical Programming was chosen, so as to increase sample variability. The "difficulty" criterion was the solution time if the instance was solved within the $30 \mathrm{~min}$ time limit, or the optimality gap attained if the solver was stopped without proving optimality. It is also possible to observe that the instances chosen come from all four quartiles, when classified using the maximum needs-to-capacity ratio (Table 19.5, in the Appendix), so as to increase diversity.

The computational tests were run on the same computer and the models were also developed and run on IBM ILOG CPLEX Optimization Studio 12.6.1.0 using the CP Optimizer. The time limit set for the resolution of each instance was also $30 \mathrm{~min}$. The eleven instances chosen and the summary of the results are presented on Table 19.2. Here, the values in bold are the best solutions found across models.

These results point to the initial hypothesis that Constraint Programming is most useful when it involves well-performing global constraints, which were able to capture and use the problem structure to propagate constraints and prune branches more

Table 19.2 Results of CP models, comparing with MIP model

\begin{tabular}{l|l|l|l|l|l|l|l|l}
\hline & \multicolumn{9}{|l}{ Objective function } & \multicolumn{2}{l}{ Solution time (s) } \\
\cline { 2 - 9 } & MIP & CP1 & CP2 & CP3 & MIP & CP1 & CP2 & CP3 \\
\hline la10 & $\mathbf{9 5 8}$ & $\mathbf{9 5 8}$ & $\mathbf{9 5 8}$ & $\mathbf{9 5 8}$ & 1.800 & 1.800 & 1.800 & 0 \\
\hline la14 & $\mathbf{1 . 2 9 2}$ & $\mathbf{1 . 2 9 2}$ & $\mathbf{1 . 2 9 2}$ & $\mathbf{1 . 2 9 2}$ & 1.800 & 1.800 & 1.800 & 0 \\
\hline orb01 & $\mathbf{1 . 0 5 9}$ & 1.096 & 1.070 & $\mathbf{1 . 0 5 9}$ & 1.013 & 1.800 & 1.800 & 28 \\
\hline la23 & $\mathbf{1 . 0 3 2}$ & $\mathbf{1 . 0 3 2}$ & $\mathbf{1 . 0 3 2}$ & $\mathbf{1 . 0 3 2}$ & 1.800 & 1.800 & 1.800 & 0 \\
\hline la30 & $\mathbf{1 . 3 5 5}$ & $\mathbf{1 . 3 5 5}$ & $\mathbf{1 . 3 5 5}$ & $\mathbf{1 . 3 5 5}$ & 1.800 & 1.800 & 1.800 & 2 \\
\hline la37 & 1.418 & 1.430 & 1.410 & $\mathbf{1 . 3 9 7}$ & 1.800 & 1.800 & 1.800 & 5 \\
\hline la34 & 1.749 & 1.721 & 1.740 & $\mathbf{1 . 7 2 1}$ & 1.800 & 1.800 & 1.800 & 1 \\
\hline abz7 & 702 & 691 & 691 & $\mathbf{6 6 3}$ & 1.800 & 1.800 & 1.800 & 1.800 \\
\hline swv10 & 2.024 & 1.915 & 1.961 & $\mathbf{1 . 8 2 7}$ & 1.800 & 1.800 & 1.800 & 1.800 \\
\hline yn4 & 1.054 & 1.044 & 1.061 & $\mathbf{9 8 6}$ & 1.800 & 1.800 & 1.800 & 1.800 \\
\hline swv13 & 4.307 & 3.829 & 3.948 & $\mathbf{3 . 1 4 9}$ & 1.800 & 1.800 & 1.800 & 1.800 \\
\hline
\end{tabular}




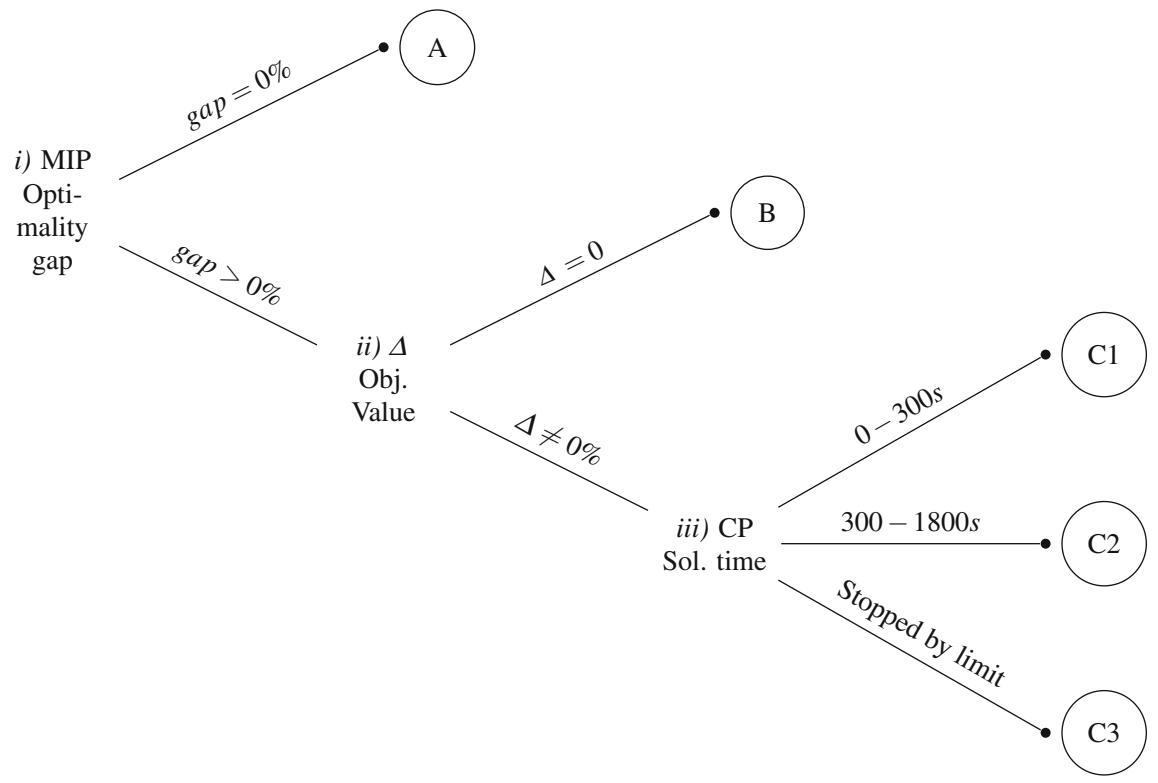

Fig. 19.5 Characteristics of the different types of instances

easily. In fact, when comparing CP1 or CP2 with the MIP model, one is able to conclude that these Constraint Programming models bring little or no advantage. As the instances get bigger, the combinations of the domains to test get exponentially large and hinder a good performance of the model. However, when the global constraints and a more appropriate representation are used (CP3), this complexity is more easily tackled and the results point to a significantly better performance of $\mathrm{CP}$ when compared with a MIP approach. Comparing the performance of CP1 and CP2 (which were very similar), it is also possible to conclude that reducing the number of constraints does not bring clear advantages, as is more usual in MIP programs.

In order to compare in more detail the Mathematical Programming and Constraint Programming approaches, the eighty-two instances were run with the full-performing CP model (CP3). The results are presented in the Appendix (Table 19.6 ${ }^{3}$ ). From the results, the instances were categorized in six categories $(\mathrm{A}, \mathrm{B}, \mathrm{C} 1, \mathrm{C} 2, \mathrm{C} 3)$, depending on three sequential criteria: $(i)$ whether or not the MIP model was able to prove the optimality of the solution found (i.e. attaining $0 \%$ optimality gap), (ii) the difference on the objective function values found by both approaches, and (iii) the run time of the CP model. Figure 19.5 depicts the instance categorization and the respective characteristics.

Instances of type A are the instances that the MIP model solved within the time limit, i.e. the optimality of the solution was proven (with $0 \%$ gap). The instances where the MIP solution optimality was not proven were divided in two groups: the

\footnotetext{
${ }^{3}$ This table also repeats the MIP results in order to facilitate the comparison instance by instance.
} 
Table 19.3 Summary of average results for CP model CP3, comparing with MIP model

\begin{tabular}{l|l|l|l|l|c}
\hline Instance type & $(\#)$ & Avg size & $\begin{array}{l}\text { Avg MIP sol. } \\
\text { time (s) }\end{array}$ & $\begin{array}{l}\text { Avg CP sol. } \\
\text { time (s) }\end{array}$ & $\begin{array}{l}\text { Avg } \Delta \text { obj. } \\
\text { value (\%) }\end{array}$ \\
\hline $\mathrm{A}$ & $(24)$ & 87 & 109 & 6 & 0 \\
\hline $\mathrm{B}$ & $(17)$ & 154 & 1.800 & 0 & 0 \\
\hline $\mathrm{C} 1$ & $(18)$ & 278 & 1.800 & 44 & 3 \\
\hline $\mathrm{C} 2$ & $(1)$ & 225 & 1.800 & 313 & -1 \\
\hline $\mathrm{C} 3$ & $(22)$ & 341 & 1.800 & 1.800 & -11 \\
\hline
\end{tabular}

instances where the solution value found by the CP Optimizer (within the time limit, hence optimal) was equal to the one attained by the MIP model (type B) and the instances where the solution values were different ${ }^{4}$ (type $C$ ). The instances of type $\mathrm{C}$ were also divided according to the solution time of the CP program. One should notice that the program was stopped by the time limit only for category $\mathrm{C} 3$; for $\mathrm{C} 1$ and C2, it stopped because it had explored all possible solutions. Table 19.3 presents the summary of the average results for each type of instance.

The average size $\mathrm{e}^{5}$ of the instances increases from A to $\mathrm{C}$, yet there is no linear relation with size and the distribution of the instances of type $\mathrm{C}$ in $\mathrm{C} 1, \mathrm{C} 2$ and $\mathrm{C} 3$. In fact, $\mathrm{C} 1$ contains both smaller instances ( 15 jobs and 5 machines) and some of the biggest instances (50 jobs and 10 machines). At the same time, other instances of the same sizes are classified as $\mathrm{C} 3$. This is an indication that also in Constraint Programming size is not a clear indicator of complexity.

Instances of type A (the smallest) were solved to optimality by both approaches, and their optimality was proven also in both cases. ${ }^{6}$ Nevertheless, the CP approach was considerably faster.

For instances of type B, since the CP Optimizer was able to explore all possible solutions under the time limit, it proved the optimality of the best solution found. As there is no difference between the solution value found by CP and MIP, it is possible to state that both approaches reached the optimum. However, although the MIP model was also able to reach the optimum for these instances, it was not able to prove so within the time limit. Considering that for these 17 instances the CP Optimizer took, in average, under a second to attain the optimal solution, this approach was able to almost instantaneously prove optimality whilst the MIP solver was not able to do so in $30 \mathrm{~min}$. This conclusion does not contrast with the notion that Constraint Programming is a technique more prone to admissibility rather than optimality: in

\footnotetext{
${ }^{4}$ I.e., there existed a delta on the objective function value given by $\Delta=\left(\right.$ ObjValue $_{C P}-$ ObjValue $_{M I P}$ )/ObjValue MI $_{P}$.

${ }^{5}$ Since the number of variables and constraints is different in the two models, size is herein considered as the number of jobs multiplied by the number of machines.

${ }^{6} \mathrm{MIP}$ model: proven by optimality gap. CP Model: the only way for a CP program to prove optimality is by exploring all possible solutions; therefore, if the solver stops before the time limit, it means that optimality was proven.
} 
fact, it was its power to efficiently check admissibility that allowed for the optimality to be proven in these cases.

As for instances of type $\mathrm{C}$, it is important to notice that there are 18 instances categorized as $\mathrm{C} 1,22$ as $\mathrm{C} 3$ yet only one as $\mathrm{C} 2$. Moreover, the instance categorized as $\mathrm{C} 2$ is close to the threshold of $\mathrm{C} 1$ (the run time is $313 \mathrm{~s}$ and the threshold is $300 \mathrm{~s}$ ). In fact, either the CP Optimizer is swift to explore all possible solutions (under $5 \mathrm{~min}$ ) or it reaches the time limit ( $30 \mathrm{~min})$ without being able to do so. Moreover, as it as mentioned above, the size is not a good indicator of this behaviour: when analysing the biggest instances (swV11-swV20), it is possible to conclude that in half of the instances the optimal solution is found in under $3 \mathrm{~s}$ while in the other half the CP Optimizer is stopped after $30 \mathrm{~min}$ without being able to explore all possible solutions. Nevertheless, there are significant improvements on the objective function value for all sub-categories of $\mathrm{C}$, especially on $\mathrm{C} 3$.

It is therefore interesting to study how swiftly the CP Optimizer finds its best solution in $30 \mathrm{~min}$. As an example, Fig. 19.6 presents the value of the incumbent solutions found throughout the run time for instance swv12. The dotted line represents the best solution found by the MIP solver for this instance (with an optimality gap of $79 \%$ ). It is possible to see that the steep improvement on the objective function value occurs in the beginning. In fact, approximately $80 \%$ of the improvement (vs. the MIP solution) occurs in $20 \mathrm{~s}$, and $95 \%$ occurs in $2 \mathrm{~min}$.

It is also interesting to analyse the swiftness to find a good solution for those instances in which both approaches were stopped by the $30 \mathrm{~min}$ time limit and where the CP presented only a slightly better performance in terms of the best objective function value found in this limit. Figure 19.7 shows the evolution of the incumbent value for both approaches for instance yn1 (20 jobs, 20 machines) in which the improvement attained by the CP approach (vs. the MIP approach) was the smallest $(1 \%)$.

As it is possible to see, the CP model was able to achieve a good solution before the MIP model. In fact, for the CP approach there was one major improvement in the

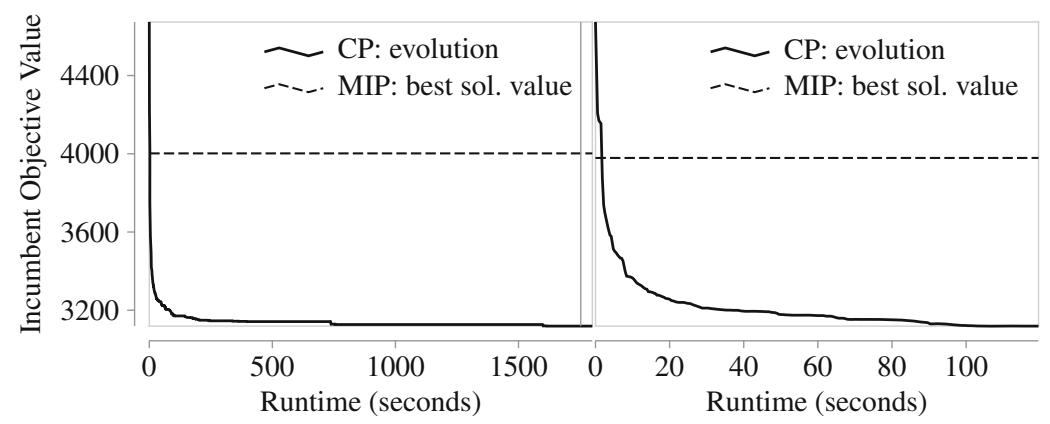

(a) Overview of the 30 minutes run time

(b) Detail of the first 2 minutes

Fig. 19.6 Evolution of the incumbent objective function value for instance swv12 throughout the 30 min run time with model CP3 
Fig. 19.7 Evolution of the incumbent objective function value for instance yn 1 throughout the $30 \mathrm{~min}$ run time with the MIP model and CP model (CP3)

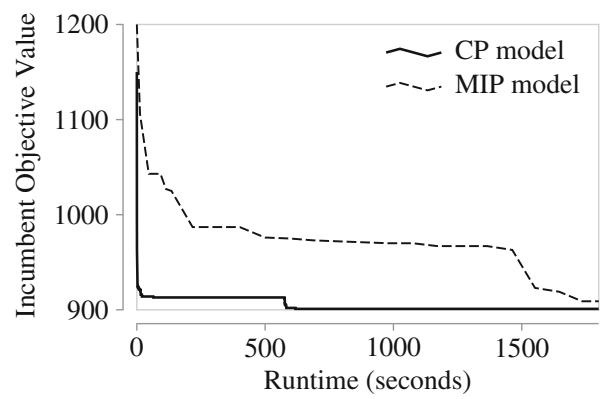

first seconds and a smaller one after approximately $10 \mathrm{~min}$. As for the MIP model, there were three major improvements: the first after a few seconds, the second at around $2-3 \mathrm{~min}$, and the third in the last $10 \mathrm{~min}$ of the time horizon. This may lead to the conclusion that this full-performing CP model is quicker than the MIP model at finding good solutions even in the case where both approaches reach the same solution within the same time limit.

One final note to highlight the fact that the needs-to-capacity ratio was not a good predictor of complexity (or "difficulty to solve") for the CP Optimizer. In fact, there was not a clear correlation between the $\mathrm{CP}$ solution time and the needs-to-capacity ratio quartile of the instances (Table 19.5, in the Appendix).

\subsection{Conclusions}

The first objective of this paper was to understand the impact of the instances' size on the intrinsic complexity of the JSSP and on its consequent "difficulty to solve". The problem was described and a MIP Model from the literature was presented and used to solve eighty-two instances. The results enabled a detailed analysis of the instances' characteristics and responsiveness to the MIP model.

From the analysis of the performance of the Mathematical Programming approach, it was possible to conclude that an instance size has a significant impact on its easiness to solve, yet it is not the only significant factor. In fact, there was significant variability on solution time for same-size instances, and for bigger instances that were not solved to optimality there was not always visible a straightforward relationship between size and optimality gap achieved.

In this approach, the size of the problem is determined by two characteristics: the number of jobs and the number of machines. From the structure of the problem and its formulation, the number of jobs has a bigger impact on the number of variables and number of constraints, hence a bigger impact on the size. Moreover, the increase on the number of jobs seems to have a straightforward relationship with the increase of optimality gap achieved. As for the number of machines, it was possible to conclude that its impact is not direct. For a specific number of jobs, it seems that an increasing 
or more balanced number of machines makes the instances easier to solve despite being of a bigger size. An hypothesis proposed to explain this behaviour is related to the easiness of the solver to find admissible integer solutions in such a problem, hence being able to update the upper bound and the optimality gap more frequently.

In order to further understand the concept of complexity, a measure of the load applied to each machine throughout the scheduling horizon was designed. The needsto-capacity ratio developed shows a significant correlation with the optimality gap attained by the MIP solver, thus being an adequate predictor of the "difficulty to solve" of an instance, for a Mathematical Programming approach.

The measure proposed, as well as the insights gained regarding the factors that induce complexity, can be useful e.g. when tackling an industrial scheduling application of the JSSP. Analysing the number of jobs and machines and other characteristics of the actual problem, it will be possible to understand whether the MIP model can easily solve the problem to optimality thus eliminating the need for more complex solution approaches or whether these are justifiably needed.

The second objective of this paper was to compare the impact of using Constraint Programming instead of Mathematical Programming to tackle the complexity of the $J S S P$. It was concluded that the main power of CP when tackling these hard, complex, combinatorial problems comes from the tailored-made global constraints that are able to swiftly improve propagation, find admissible solutions and thus significantly decrease the solution time. In fact, the CP models that used the same variables as the MIP model and similar constraints - which benefited only from part of the CP modelling flexibility by the use of logic and non-linear constraints - performed as well as, or worse than, the MIP model for the instances tested. The CP model that used a different representation of the variables and solid global constraints, however, was able to get significant improvements either in solution time and quality of the solutions found.

It was also possible to conclude that for some instances, although the MIP solver presented high optimality gap values at the end of the time limit, it was indeed able to reach the optimum. Nevertheless, it was not able to prove so. This conclusion is built on the fact that, within seconds, the (well-performing) CP model was able to test all possible admissible solutions and state that said solution was the best possible. Moreover, it was possible to conclude that the instance size has a significantly smaller impact in Constraint Programming than in Mathematical Programming. In fact, half of the largest instances tested were solved to optimality in a few seconds while the run of the remaining half was stopped by the $30 \mathrm{~min}$ time limit. There were also indicators that the full-performing CP approach may be swifter than the MIP approach at finding good solutions, even when it is not able to prove optimality in the specified time limit and it is not able to attain major improvements on the best solution value found.

Since the best performing CP model, which presents all the above-mentioned advantages, is commercially available, these conclusions may have a significant impact on practical application of optimization methods. In fact, it is pointed out that there are Constraint Programming models available for this type of problem that may be a better alternative to the "traditional" MIP approach for some problems. 
Future work will be focused on comparing other characteristics of both approaches, such as the number of feasible solutions found in a specific time frame, which might be useful when using hybrid solution methods such as matheuristics to solve this problem. Additionally, it would be interesting to study the reasons why the needsto-capacity ratio does not appear to be a good predictor of "difficulty to solve" for CP models.

Acknowledgements The first author was supported by grant SFRH/BD/103362/2014 from FCT Fundação para a Ciência e Tecnologia (Portuguese Foundation for Science and Technology). This work was also partially financed by the ERDF - European Regional Development Fund through the Operational Programme for Competitiveness and Internationalisation - COMPETE 2020 Programme within project "POCI-01-0145-FEDER-006961", and by National Funds through the FCT - Fundação para a Ciência e Tecnologia (Portuguese Foundation for Science and Technology) as part of project UID/EEA/50014/2013.

\section{Appendix}

See Tables 19.4, 19.5 and 19.6.

Table 19.4 Size and characteristics of the instances run and corresponding results with the MIP model

\begin{tabular}{l|l|l|l|l|l}
\hline Instance name & Jobs & Machines & Obj. function value & Solution time (s) & Optimality gap (\%) \\
\hline $\mathrm{ft} 06$ & 6 & 6 & 55 & 0 & 0 \\
\hline $\mathrm{abz5}$ & 10 & 10 & 1.234 & 19 & 0 \\
\hline $\mathrm{abz6}$ & 10 & 10 & 943 & 4 & 0 \\
\hline $\mathrm{ft} 10$ & 10 & 10 & 930 & 122 & 0 \\
\hline $\mathrm{la} 01$ & 10 & 5 & 666 & 7 & 0 \\
\hline $\mathrm{la} 02$ & 10 & 5 & 655 & 6 & 0 \\
\hline $\mathrm{la} 03$ & 10 & 5 & 597 & 3 & 0 \\
\hline $\mathrm{la} 04$ & 10 & 5 & 590 & 2 & 0 \\
\hline la05 & 10 & 5 & 593 & 45 & 0 \\
\hline la16 & 10 & 10 & 945 & 7 & 0 \\
\hline la17 & 10 & 10 & 784 & 13 & 0 \\
\hline la18 & 10 & 10 & 848 & 3 & 0 \\
\hline la19 & 10 & 10 & 842 & 6 & 0 \\
\hline la20 & 10 & 10 & 902 & 4 & 0 \\
\hline orb01 & 10 & 10 & 1.059 & 1.013 & 0 \\
\hline orb02 & 10 & 10 & 888 & 9 & 0 \\
\hline & & & & &
\end{tabular}


Table 19.4 (continued)

\begin{tabular}{|c|c|c|c|c|c|}
\hline Instance name & Jobs & Machines & Obj. function value & Solution time (s) & Optimality gap (\%) \\
\hline orb03 & 10 & 10 & 1.005 & 590 & 0 \\
\hline orb04 & 10 & 10 & 1.005 & 38 & 0 \\
\hline orb05 & 10 & 10 & 887 & 17 & 0 \\
\hline orb06 & 10 & 10 & 1.010 & 596 & 0 \\
\hline orb07 & 10 & 10 & 397 & 14 & 0 \\
\hline orb08 & 10 & 10 & 899 & 36 & 0 \\
\hline orb09 & 10 & 10 & 934 & 56 & 0 \\
\hline orb10 & 10 & 10 & 944 & 14 & 0 \\
\hline la06 & 15 & 5 & 926 & 1.800 & 33.91 \\
\hline la07 & 15 & 5 & 890 & 1.800 & 26.07 \\
\hline la08 & 15 & 5 & 863 & 1.800 & 26.66 \\
\hline la09 & 15 & 5 & 951 & 1.800 & 25.00 \\
\hline la10 & 15 & 5 & 958 & 1.800 & 34.66 \\
\hline la21 & 15 & 10 & 1.071 & 1.800 & 15.07 \\
\hline $1 \mathrm{a} 22$ & 15 & 10 & 932 & 1.800 & 7.41 \\
\hline la23 & 15 & 10 & 1.032 & 1.800 & 23.42 \\
\hline la24 & 15 & 10 & 940 & 1.800 & 4.68 \\
\hline la25 & 15 & 10 & 979 & 1.800 & 6.54 \\
\hline la36 & 15 & 15 & 1.281 & 1.800 & 10.54 \\
\hline la37 & 15 & 15 & 1.418 & 1.800 & 15.31 \\
\hline la38 & 15 & 15 & 1.207 & 1.800 & 11.76 \\
\hline la39 & 15 & 15 & 1.240 & 1.800 & 8.55 \\
\hline la40 & 15 & 15 & 1.245 & 1.800 & 14.78 \\
\hline abz7 & 20 & 15 & 702 & 1.800 & 31.45 \\
\hline abz8 & 20 & 15 & 715 & 1.800 & 28.95 \\
\hline abz9 & 20 & 15 & 721 & 1.800 & 27.04 \\
\hline $\mathrm{ft} 20$ & 20 & 5 & 1.198 & 1.800 & 48.51 \\
\hline la11 & 20 & 5 & 1.222 & 1.800 & 50.31 \\
\hline la12 & 20 & 5 & 1.039 & 1.800 & 40.69 \\
\hline la13 & 20 & 5 & 1.150 & 1.800 & 41.91 \\
\hline la14 & 20 & 5 & 1.292 & 1.800 & 52.46 \\
\hline la15 & 20 & 5 & 1.207 & 1.800 & 49.59 \\
\hline la26 & 20 & 10 & 1.218 & 1.800 & 26.93 \\
\hline la27 & 20 & 10 & 1.288 & 1.800 & 33.39 \\
\hline la28 & 20 & 10 & 1.224 & 1.800 & 31.74 \\
\hline la29 & 20 & 10 & 1.220 & 1.800 & 26.42 \\
\hline la30 & 20 & 10 & 1.355 & 1.800 & 34.64 \\
\hline
\end{tabular}


19 Understanding Complexity in a Practical Combinatorial Problem ...

Table 19.4 (continued)

\begin{tabular}{|c|c|c|c|c|c|}
\hline Instance name & Jobs & Machines & Obj. function value & Solution time (s) & Optimality gap (\%) \\
\hline swv01 & 20 & 10 & 1.563 & 1.800 & 46.45 \\
\hline swv02 & 20 & 10 & 1.579 & 1.800 & 45.40 \\
\hline swv03 & 20 & 10 & 1.618 & 1.800 & 47.42 \\
\hline swv04 & 20 & 10 & 1.625 & 1.800 & 48.51 \\
\hline swv05 & 20 & 10 & 1.633 & 1.800 & 45.81 \\
\hline swv06 & 20 & 15 & 1.868 & 1.800 & 40.26 \\
\hline swv07 & 20 & 15 & 1.736 & 1.800 & 38.28 \\
\hline swv08 & 20 & 15 & 2.046 & 1.800 & 41.88 \\
\hline swv09 & 20 & 15 & 1.907 & 1.800 & 41.85 \\
\hline swv10 & 20 & 15 & 2.024 & 1.800 & 45.65 \\
\hline yn1 & 20 & 20 & 913 & 1.800 & 17.50 \\
\hline yn2 & 20 & 20 & 954 & 1.800 & 21.91 \\
\hline yn3 & 20 & 20 & 945 & 1.800 & 20.33 \\
\hline yn4 & 20 & 20 & 1.054 & 1.800 & 27.42 \\
\hline la31 & 30 & 10 & 1.784 & 1.800 & 55.77 \\
\hline la32 & 30 & 10 & 1.850 & 1.800 & 52.68 \\
\hline la33 & 30 & 10 & 1.719 & 1.800 & 54.53 \\
\hline la34 & 30 & 10 & 1.749 & 1.800 & 57.72 \\
\hline la35 & 30 & 10 & 1.888 & 1.800 & 55.58 \\
\hline swv11 & 50 & 10 & 3.767 & 1.800 & 77.84 \\
\hline swv12 & 50 & 10 & 4.002 & 1.800 & 78.68 \\
\hline swv13 & 50 & 10 & 4.307 & 1.800 & 80.37 \\
\hline swv14 & 50 & 10 & 4.081 & 1.800 & 79.66 \\
\hline swv15 & 50 & 10 & 3.722 & 1.800 & 78.02 \\
\hline swv16 & 50 & 10 & 2.952 & 1.800 & 73.41 \\
\hline swv17 & 50 & 10 & 2.915 & 1.800 & 72.10 \\
\hline swv18 & 50 & 10 & 2.950 & 1.800 & 74.16 \\
\hline 0swv19 & 50 & 10 & 3.107 & 1.800 & 74.67 \\
\hline swv20 & 50 & 10 & 3.009 & 1.800 & 74.46 \\
\hline \multicolumn{3}{|c|}{ abz5 - abz9 in Adams et al. [2] } & \multicolumn{3}{|c|}{$\mathrm{ft} 06, \mathrm{ft} 10, \mathrm{ft} 20$ in Fisher and Thompson [6] } \\
\hline \multicolumn{3}{|c|}{ la01 - la40 in Lawrence [7] } & \multicolumn{3}{|c|}{ orb01 - orb10 in Applegate and Cook [3] } \\
\hline \multicolumn{3}{|c|}{ swv01 - swv20 in Storer et al. [11] } & \multicolumn{3}{|c|}{ yn1 - yn4 in Yamada and Nakano [14] } \\
\hline
\end{tabular}


Table 19.5 Needs-to-capacity ratio calculation for each instance

\begin{tabular}{|c|c|c|c|c|c|c|}
\hline \multicolumn{7}{|c|}{ Needs-to-capacity ratio } \\
\hline $\begin{array}{l}\text { Instance } \\
\text { name }\end{array}$ & $\begin{array}{l}\text { Optimality } \\
\text { gap (\%) }\end{array}$ & $\begin{array}{l}\text { Maximum } \\
\text { value }\end{array}$ & $\begin{array}{l}\text { Quartile } \\
\text { (of max) }\end{array}$ & $\begin{array}{l}\text { Std. } \\
\text { deviation }\end{array}$ & \# partitions & \begin{tabular}{|l|} 
Partition \\
length
\end{tabular} \\
\hline $\mathrm{ft} 06$ & 0 & 1.17 & $1 \mathrm{st}$ & 0.30 & 27 & 2 \\
\hline abz5 & 0 & 2.00 & 3rd & 0.55 & 9 & 100 \\
\hline abz6 & 0 & 1.20 & 2nd & 0.34 & 18 & 40 \\
\hline $\mathrm{ft} 10$ & 0 & 1.00 & $1 \mathrm{st}$ & 0.11 & 159 & 4 \\
\hline la01 & 0 & 2.60 & $3 \mathrm{rd}$ & 0.63 & 17 & 24 \\
\hline $1 \mathrm{a} 02$ & 0 & 2.40 & 3rd & 0.64 & 15 & 24 \\
\hline la03 & 0 & 2.00 & 3rd & 0.43 & 31 & 14 \\
\hline $1 \mathrm{a} 04$ & 0 & 2.00 & $3 \mathrm{rd}$ & 0.35 & 42 & 10 \\
\hline $\mathrm{la} 05$ & 0 & 2.20 & 3rd & 0.39 & 39 & 10 \\
\hline la16 & 0 & 1.00 & $1 \mathrm{st}$ & 0.19 & 51 & 14 \\
\hline la17 & 0 & 1.00 & $1 \mathrm{st}$ & 0.17 & 67 & 10 \\
\hline la18 & 0 & 1.00 & $1 \mathrm{st}$ & 0.17 & 65 & 10 \\
\hline la19 & 0 & 1.00 & 1st & 0.19 & 56 & 10 \\
\hline la20 & 0 & 1.20 & 2nd & 0.20 & 60 & 12 \\
\hline orb01 & 0 & 1.10 & 1st & 0.18 & 68 & 10 \\
\hline orb02 & 0 & 1.00 & $1 \mathrm{st}$ & 0.16 & 50 & 12 \\
\hline orb03 & 0 & 1.00 & $1 \mathrm{st}$ & 0.16 & 66 & 10 \\
\hline orb04 & 0 & 1.10 & 1st & 0.17 & 76 & 10 \\
\hline orb05 & 0 & 1.10 & 1st & 0.18 & 55 & 10 \\
\hline orb06 & 0 & 1.00 & 1st & 0.19 & 61 & 12 \\
\hline orb07 & 0 & 1.20 & 2nd & 0.25 & 28 & 10 \\
\hline orb08 & 0 & 1.20 & 2nd & 0.18 & 66 & 10 \\
\hline orb09 & 0 & 1.00 & 1st & 0.17 & 69 & 10 \\
\hline orb10 & 0 & 1.10 & 1st & 0.17 & 78 & 10 \\
\hline la06 & 34 & 3.00 & 3rd & 0.63 & 29 & 14 \\
\hline la07 & 26 & 3.60 & 4th & 0.77 & 25 & 16 \\
\hline la08 & 27 & 3.40 & 4th & 0.59 & 38 & 10 \\
\hline la09 & 25 & 3.40 & 4th & 0.64 & 26 & 14 \\
\hline la10 & 35 & 3.00 & $3 \mathrm{rd}$ & 0.51 & 44 & 10 \\
\hline la21 & 15 & 1.70 & 2nd & 0.28 & 48 & 14 \\
\hline la22 & 7 & 1.50 & 2nd & 0.23 & 59 & 10 \\
\hline la23 & 23 & 1.60 & 2nd & 0.23 & 73 & 10 \\
\hline la24 & 5 & 1.70 & 2nd & 0.24 & 75 & 10 \\
\hline la25 & 7 & 1.70 & 2nd & 0.25 & 77 & 10 \\
\hline la36 & 11 & 1.00 & $1 \mathrm{st}$ & 0.17 & 69 & 14 \\
\hline la37 & 15 & 1.00 & $1 \mathrm{st}$ & 0.15 & 95 & 10 \\
\hline la38 & 12 & 1.13 & 1st & 0.15 & 98 & 10 \\
\hline
\end{tabular}


19 Understanding Complexity in a Practical Combinatorial Problem ...

Table 19.5 (continued)

Needs-to-capacity ratio

\begin{tabular}{|c|c|c|c|c|c|c|}
\hline $\begin{array}{l}\text { Instance } \\
\text { name }\end{array}$ & $\begin{array}{l}\text { Optimality } \\
\text { gap (\%) }\end{array}$ & $\begin{array}{l}\text { Maximum } \\
\text { value }\end{array}$ & $\begin{array}{l}\text { Quartile (of } \\
\text { max) }\end{array}$ & \begin{tabular}{|l|} 
Std. \\
deviation
\end{tabular} & \# partitions & $\begin{array}{l}\text { Partition } \\
\text { length }\end{array}$ \\
\hline la39 & 9 & 1.00 & $1 \mathrm{st}$ & 0.15 & 95 & 10 \\
\hline la40 & 15 & 1.00 & $1 \mathrm{st}$ & 0.15 & 91 & 10 \\
\hline abz7 & 31 & 1.73 & 2nd & 0.41 & 19 & 22 \\
\hline abz8 & 29 & 2.27 & $3 \mathrm{rd}$ & 0.53 & 21 & 22 \\
\hline abz9 & 27 & 1.80 & 2nd & 0.54 & 23 & 22 \\
\hline $\mathrm{ft} 20$ & 49 & 4.20 & 4th & 0.44 & 111 & 4 \\
\hline la11 & 50 & 4.60 & 4th & 0.90 & 31 & 14 \\
\hline la12 & 41 & 4.40 & 4 th & 0.76 & 41 & 10 \\
\hline la13 & 42 & 4.00 & 4th & 0.71 & 42 & 10 \\
\hline la14 & 52 & 4.40 & 4th & 0.72 & 45 & 10 \\
\hline la15 & 50 & 4.40 & 4th & 0.80 & 32 & 12 \\
\hline $1 \mathrm{a} 26$ & 27 & 2.20 & $3 \mathrm{rd}$ & 0.36 & 50 & 14 \\
\hline la27 & 33 & 2.00 & $3 \mathrm{rd}$ & 0.27 & 64 & 10 \\
\hline la28 & 32 & 2.10 & $3 \mathrm{rd}$ & 0.31 & 81 & 10 \\
\hline la29 & 26 & 2.20 & $3 r d$ & 0.31 & 76 & 10 \\
\hline la30 & 35 & 2.00 & $3 \mathrm{rd}$ & 0.30 & 77 & 10 \\
\hline swv01 & 46 & 2.00 & $3 \mathrm{rd}$ & 0.14 & 356 & 2 \\
\hline swv02 & 45 & 2.00 & $3 \mathrm{rd}$ & 0.15 & 330 & 2 \\
\hline swv03 & 47 & 2.20 & $3 \mathrm{rd}$ & 0.15 & 334 & 2 \\
\hline swv04 & 49 & 2.10 & $3 \mathrm{rd}$ & 0.15 & 351 & 2 \\
\hline swv05 & 46 & 2.10 & $3 \mathrm{rd}$ & 0.14 & 390 & 2 \\
\hline swv06 & 40 & 1.40 & 2nd & 0.09 & 483 & 2 \\
\hline swv07 & 38 & 1.33 & 2nd & 0.09 & 449 & 2 \\
\hline swv08 & 42 & 1.40 & 2nd & 0.09 & 527 & 2 \\
\hline swv09 & 42 & 1.40 & 2nd & 0.09 & 476 & 2 \\
\hline swv10 & 46 & 1.40 & 2nd & 0.13 & 231 & 4 \\
\hline yn1 & 17 & 1.30 & 2nd & 0.23 & 33 & 20 \\
\hline yn2 & 22 & 1.40 & 2nd & 0.31 & 37 & 20 \\
\hline yn3 & 20 & 1.15 & $1 \mathrm{st}$ & 0.28 & 35 & 20 \\
\hline yn4 & 27 & 1.20 & 2nd & 0.27 & 37 & 20 \\
\hline la31 & 56 & 3.30 & 4th & 0.46 & 73 & 10 \\
\hline la32 & 53 & 3.10 & $3 \mathrm{rd}$ & 0.43 & 79 & 10 \\
\hline la33 & 55 & 3.30 & 4th & 0.46 & 75 & 10 \\
\hline la34 & 58 & 3.00 & $3 \mathrm{rd}$ & 0.43 & 60 & 10 \\
\hline la35 & 56 & 3.10 & 3rd & 0.45 & 68 & 10 \\
\hline swv11 & 78 & 5.00 & 4th & 0.30 & 397 & 2 \\
\hline swv12 & 79 & 5.30 & 4th & 0.31 & 390 & 2 \\
\hline
\end{tabular}


Table 19.5 (continued)

\begin{tabular}{l|l|l|l|l|l|l}
\hline $\begin{array}{l}\text { Needs-to-capacity ratio } \\
\begin{array}{l}\text { Instance } \\
\text { name }\end{array}\end{array}$ & $\begin{array}{l}\text { Optimality } \\
\text { gap (\%) }\end{array}$ & $\begin{array}{l}\text { Maximum } \\
\text { value }\end{array}$ & $\begin{array}{l}\text { Quartile (of } \\
\text { max })\end{array}$ & $\begin{array}{l}\text { Std. } \\
\text { deviation }\end{array}$ & \# partitions & $\begin{array}{l}\text { Partition } \\
\text { length }\end{array}$ \\
\hline swv13 & 80 & 5.20 & 4th & 0.31 & 382 & 2 \\
\hline swv14 & 80 & 5.30 & 4th & 0.31 & 382 & 2 \\
\hline swv15 & 78 & 5.00 & 4th & 0.32 & 339 & 2 \\
\hline swv16 & 73 & 5.10 & 4th & 0.31 & 372 & 2 \\
\hline swv17 & 72 & 5.00 & 4th & 0.33 & 308 & 2 \\
\hline swv18 & 74 & 5.00 & 4th & 0.32 & 331 & 2 \\
\hline swv19 & 75 & 5.00 & 4th & 0.31 & 339 & 2 \\
\hline swv20 & 74 & 5.10 & 4th & 0.32 & 333 & 2 \\
\hline
\end{tabular}

Table 19.6 Instances run (with $J$ jobs and $M$ machines) with MIP model and CP model CP3

\begin{tabular}{l|l|l|l|l|l}
\hline Instance & $\begin{array}{l}\text { MIP optimality } \\
\text { gap }(\%)\end{array}$ & $\begin{array}{l}\text { MIP obj. } \\
\text { function value }\end{array}$ & $\begin{array}{l}\text { CP3 Obj. } \\
\text { function value }\end{array}$ & $\begin{array}{l}\text { MIP sol. } \\
\text { time (s) }\end{array}$ & $\begin{array}{l}\text { CP3 sol. } \\
\text { time (s) }\end{array}$ \\
\hline ft06 & 0.0 & 55 & 55 & 0 & 0 \\
\hline abz5 & 0.0 & 1.234 & 1.234 & 19 & 7 \\
\hline abz6 & 0.0 & 943 & 943 & 4 & 2 \\
\hline ft10 & 0.0 & 930 & 930 & 122 & 12 \\
\hline la01 & 0.0 & 666 & 666 & 7 & 0 \\
\hline la02 & 0.0 & 655 & 655 & 6 & 0 \\
\hline la03 & 0.0 & 597 & 597 & 3 & 0 \\
\hline la04 & 0.0 & 590 & 590 & 2 & 1 \\
\hline la05 & 0.0 & 593 & 593 & 45 & 0 \\
\hline la16 & 0.0 & 945 & 945 & 7 & 2 \\
\hline la17 & 0.0 & 784 & 784 & 13 & 2 \\
\hline la18 & 0.0 & 848 & 848 & 3 & 2 \\
\hline la19 & 0.0 & 842 & 842 & 6 & 10 \\
\hline la20 & 0.0 & 902 & 902 & 4 & 3 \\
\hline orb01 & 0.0 & 1.059 & 1.059 & 1.013 & 28 \\
\hline orb02 & 0.0 & 888 & 888 & 9 & 7 \\
\hline orb03 & 0.0 & 1.005 & 1.005 & 590 & 19 \\
\hline orb04 & 0.0 & 1.005 & 1.005 & 38 & 10 \\
\hline orb05 & 0.0 & 887 & 887 & 17 & 7 \\
\hline orb06 & 0.0 & 1.010 & 1.010 & 596 & 13 \\
\hline orb07 & 0.0 & 397 & 397 & 14 & 4 \\
\hline orb08 & 0.0 & 899 & 899 & 36 & 5 \\
\hline orb09 & 0.0 & 934 & 934 & 56 & 14 \\
\hline orb10 & 0.0 & 944 & 944 & & 3 \\
\hline & & & & 74 & \\
\hline
\end{tabular}


19 Understanding Complexity in a Practical Combinatorial Problem ...

Table 19.6 (continued)

\begin{tabular}{|c|c|c|c|c|c|}
\hline Instance & $\begin{array}{l}\text { MIP optimality } \\
\text { gap }(\%)\end{array}$ & $\begin{array}{l}\text { MIP obj. } \\
\text { function value }\end{array}$ & $\begin{array}{l}\text { CP3 Obj. } \\
\text { function value }\end{array}$ & $\begin{array}{l}\text { MIP sol. } \\
\text { time (s) }\end{array}$ & $\begin{array}{l}\text { CP3 sol. } \\
\text { time (s) }\end{array}$ \\
\hline la06 & 33.9 & 926 & 926 & 1.800 & 0 \\
\hline la07 & 26.1 & 890 & 890 & 1.800 & 0 \\
\hline la08 & 26.7 & 863 & 863 & 1.800 & 0 \\
\hline la09 & 25.0 & 951 & 951 & 1.800 & 0 \\
\hline la10 & 34.7 & 958 & 958 & 1.800 & 0 \\
\hline la21 & 15.1 & 1.071 & 1.046 & 1.800 & 76 \\
\hline $1 a 22$ & 7.4 & 932 & 927 & 1.800 & 9 \\
\hline la23 & 23.4 & 1.032 & 1.032 & 1.800 & 0 \\
\hline la24 & 4.7 & 940 & 935 & 1.800 & 35 \\
\hline la25 & 6.5 & 979 & 977 & 1.800 & 52 \\
\hline la36 & 10.5 & 1.281 & 1.268 & 1.800 & 13 \\
\hline la37 & 15.3 & 1.418 & 1.397 & 1.800 & 5 \\
\hline la38 & 11.8 & 1.207 & 1.196 & 1.800 & 313 \\
\hline la39 & 8.5 & 1.240 & 1.233 & 1.800 & 18 \\
\hline la40 & 14.8 & 1.245 & 1.222 & 1.800 & 82 \\
\hline abz7 & 31.5 & 702 & 663 & 1.800 & 1.800 \\
\hline abz8 & 29.0 & 715 & 680 & 1.800 & 1.800 \\
\hline abz9 & 27.0 & 721 & 686 & 1.800 & 1.800 \\
\hline $\mathrm{ft} 20$ & 48.5 & 1.198 & 1.165 & 1.800 & 1 \\
\hline la11 & 50.3 & 1.222 & 1.222 & 1.800 & 0 \\
\hline la12 & 40.7 & 1.039 & 1.039 & 1.800 & 0 \\
\hline la13 & 41.9 & 1.150 & 1.150 & 1.800 & 0 \\
\hline la14 & 52.5 & 1.292 & 1.292 & 1.800 & 0 \\
\hline la15 & 49.6 & 1.207 & 1.207 & 1.800 & 0 \\
\hline la26 & 26.9 & 1.218 & 1.218 & 1.800 & 1 \\
\hline la27 & 33.4 & 1.288 & 1.235 & 1.800 & 254 \\
\hline la28 & 31.7 & 1.224 & 1.216 & 1.800 & 31 \\
\hline la29 & 26.4 & 1.220 & 1.153 & 1.800 & 1.800 \\
\hline la30 & 34.6 & 1.355 & 1.355 & 1.800 & 2 \\
\hline swv01 & 46.4 & 1.563 & 1.413 & 1.800 & 1.800 \\
\hline swv02 & 45.4 & 1.579 & 1.475 & 1.800 & 203 \\
\hline swv03 & 47.4 & 1.618 & 1.406 & 1.800 & 1.800 \\
\hline swv04 & 48.5 & 1.625 & 1.488 & 1.800 & 1.800 \\
\hline swv05 & 45.8 & 1.633 & 1.438 & 1.800 & 1.800 \\
\hline swv06 & 40.3 & 1.868 & 1.710 & 1.800 & 1.800 \\
\hline swv07 & 38.3 & 1.736 & 1.667 & 1.800 & 1.800 \\
\hline swv08 & 41.9 & 2.046 & 1.810 & 1.800 & 1.800 \\
\hline swv09 & 41.8 & 1.907 & 1.698 & 1.800 & 1.800 \\
\hline
\end{tabular}


Table 19.6 (continued)

\begin{tabular}{l|l|l|l|l|l}
\hline Instance & $\begin{array}{l}\text { MIP optimality } \\
\text { gap }(\%)\end{array}$ & $\begin{array}{l}\text { MIP obj. } \\
\text { function value }\end{array}$ & $\begin{array}{l}\text { CP3 Obj. } \\
\text { function value }\end{array}$ & $\begin{array}{l}\text { MIP sol. } \\
\text { time (s) }\end{array}$ & $\begin{array}{l}\text { CP3 sol. } \\
\text { time (s) }\end{array}$ \\
\hline swv10 & 45.7 & 2.024 & 1.827 & 1.800 & 1.800 \\
\hline yn1 & 17.5 & 913 & 901 & 1.800 & 1.800 \\
\hline yn2 & 21.9 & 954 & 910 & 1.800 & 1.800 \\
\hline yn3 & 20.3 & 945 & 910 & 1.800 & 1.800 \\
\hline yn4 & 27.4 & 1.054 & 986 & 1.800 & 1.800 \\
\hline la31 & 55.8 & 1.784 & 1.784 & 1.800 & 1 \\
\hline la32 & 52.7 & 1.850 & 1.850 & 1.800 & 0 \\
\hline la33 & 54.5 & 1.719 & 1.719 & 1.800 & 0 \\
\hline la34 & 57.7 & 1.749 & 1.721 & 1.800 & 1 \\
\hline la35 & 55.6 & 1.888 & 1.888 & 1.800 & 1 \\
\hline swv11 & 77.8 & 3.767 & 3.021 & 1.800 & 1.800 \\
\hline swv12 & 78.7 & 4.002 & 3.119 & 1.800 & 1.800 \\
\hline swv13 & 80.4 & 4.307 & 3.149 & 1.800 & 1.800 \\
\hline swv14 & 79.7 & 4.081 & 2.970 & 1.800 & 1.800 \\
\hline swv15 & 78.0 & 3.722 & 2.960 & 1.800 & 1.800 \\
\hline swv16 & 73.4 & 2.952 & 2.924 & 1.800 & 0 \\
\hline swv17 & 72.1 & 2.915 & 2.794 & 1.800 & 0 \\
\hline swv18 & 74.2 & 2.950 & 2.852 & 1.800 & 0 \\
\hline swv19 & 74.7 & 3.107 & 2.843 & 1.800 & 2 \\
\hline swv20 & 74.5 & 3.009 & 2.823 & 1.800 & 0 \\
\hline & & & & &
\end{tabular}

\section{References}

1. 4COutreachProgram. CSP tutorial (2005), http://4c.ucc.ie/web/outreach/tutorial.html

2. J. Adams, E. Balas, D. Zawack, The shifting bottleneck procedure for job shop scheduling. Manag. Sci. 34, 391-401 (1988)

3. D. Applegate, W. Cook, A computational study of the job-shop scheduling instance. ORSA J. Comput. 3, 149-156 (1991)

4. K.R. APT, Priciples of Constraint Programming (Cambridge University Press, Cambridge, 2003)

5. E. Balas, Disjunctive programming. Ann. Discret. Math. 5, 3-51 (1979)

6. H. Fisher, G.L. Thompson, Probabilistic learning combinations of local job-shop scheduling rules, in Industrial Scheduling, ed. by J.F. Muth, G.L. Thompson (Prentice-Hall, Englewood Cliffs, 1963), pp. 225-251

7. S. Lawrence, Resource constrained project scheduling: an experimental investigation of heuristic scheduling techniques (supplement), in Graduate School of Industrial Administration, Carnegie-Mellon University, Pittsburgh, Pennsylvania (1984)

8. J.K. Lenstra, Computational complexity of discrete optimization problems. Ann. Discret. Math. 4, 121-140 (1979)

9. J. Poppenborg, S. Knust, J. Hertzber, Online scheduling of flexible job-shops with blocking and transportation. Eur. J. Ind. Eng. 6, 497-518 (2012) 
10. F. Rossi, P. van Beek, T. Walsh (eds.), Handbook of Constraint Programming (Elsevier, Amsterdam, 2006)

11. R.H. Storer, S.D. Wu, R. Vaccari, New search spaces for sequencing instances with application to job shop scheduling. Manag. Sci. 38, 1495-1509 (1992)

12. K. Thörnblad, A.B. Strömberg, M. Patriksson, T. Almgren, Scheduling optimisation of a real flexible job shop including fixture availability and preventive maintenance. Eur. J. Ind. Eng. 9, 126-145 (2015)

13. W.J. van Hoeve, Introduction to constraint programming, in ACP Summer School on Theory and Practice of Constraint Programming, September 24-28, 2012, Wrocław, Poland (2012)

14. T. Yamada, R. Nakano, A genetic algorithm applicable to large-scale job-shop instances, in PPSN'2 Proceedings of the 2nd International Workshop on Parallel Problem Solving from Nature, ed. by R. Manner, B. Manderick (1992), pp. 281-290 